\title{
Influencia del nivel socioeconómico (N SE) en el asma bronquial y cambios en su prevalencia en población escolar en un periodo de 6 años
}

\author{
G onzalo Valdivia $C^{1}$, Solange Caussade $L^{2}$, \\ Héctor $N$ avarro $M^{2}$, Jaime Cerda $L^{1}$, Enrique Pérez $B^{a}$, \\ Andrés Aquevedo $\mathrm{S}^{\mathrm{a}}$, Ignacio Sánchez $\mathrm{D}^{2}$.
}

\section{Changes in asthma prevalence among school children during a 6 -year period: Influence of socioeconomic status}

Background: Asthma prevalence is increasing worldwide, especially in developing countries. Aim: To determine the prevalence of asthma diagnosis and related symptoms in children, its changes during a 6-year period and the influence exerted by socioeconomic status. Materials and methods: Cross-sectional study conducted among school children of Santiago. A sample of 4,561 children aged 6-7 and 13-14 years attending public schools (central district, medium-low and low socioeconomic status) and private schools (eastern districts, high socioecomic status) were surveyed. Dependent variables were asthma diagnosis (AD), wheezing during the last 12 months (WHE12) and frequency of episodes of wheezing (FEW). Prevalence odds ratios were calculated using regression models, adjusted for several confounding variables. Results: $\mathrm{AD}$ prevalence for the group as a whole was higher in boys than in girls $(13.2 \%$ and $10.8 \%$, respectively, $\mathrm{p}=0.016)$. Prevalence rate of $\mathrm{AD}$ was also higher for children aged $13-14$ than $6-7$ years $(13.8 \%$ and $10.1 \%$, respectively $\mathrm{p}<0.01)$. An inverse association was found between SES and WHE12. Higher maternal education level was associated with lower prevalence of WHE12 in both age groups and with lower prevalence of FEW in 6-7 year-old children. Paternal education level was positively associated with $\mathrm{AD}$ among younger children. At the central district, increasing trends of WHE12 and FEW prevalence were observed during 1994-2000 among 6-7 years children. Conclusions: A significant association was observed between socioeconomic status, asthma diagnosis and symptoms in school children living in Santiago. Data support an increasing trend of asthma prevalence and severity among 6-7 year-old children during the period 1994-2000 (Rev Méd Chile 2009; 137: 215-25).

(Key words: Asthma; Children; Socioeconomic factors)

Recibido el 11 de agosto, 2008. Aceptado el 23 de octubre, 2008.

${ }^{1}$ Departamento de Salud Pública, ${ }^{2}$ Sección Respiratorio Pediátrico, Departamento de Pediatría, Facultad de Medicina, Pontificia Universidad Católica de Chile, Santiago de Chile.

aAyudante alumno, Salud Pública, Facultad de Medicina, Pontificia Universidad Católica de Chile.

Correspondencia a: Dr. Gonzalo Valdivia C. Departamento de Salud Pública, Pontificia Universidad Católica de Chile. Marcoleta 434, Santiago Centro, Santiago-Chile. Tel.: (56-2) 3543038. Fax: (56-2) 633-1840. E mail: valdivia@med.puc.cl 
$\mathrm{L}$ a prevalencia de asma ha aumentado en países desarrollados y en vías de desarrollo, siendo las consecuencias económicas y en calidad de vida derivadas de la enfermedad mayores en los últimos ${ }^{1}$. Este aumento se explica parcialmente por elementos asociados con atopía, siendo aún desconocidas las causas últimas de este fenómeno. En los últimos años se ha medido la prevalencia de síntomas respiratorios en población escolar, especialmente en países desarrollados, contándose con escasa información en América Latina ${ }^{2-4}$. Estudios chilenos en escolares entregan algunas cifras de referencia, siendo sus resultados de limitado valor para establecer comparaciones debido al uso de metodologías no estandarizadas ${ }^{5,6}$.

Diversas investigaciones reportan mayor prevalencia de asma y atopía en países occidentales, observándose un fenómeno similar en aquellos que adquieren este status en breves periodos de tiempo $^{7}$. Estudios ecológicos sugieren una relación positiva entre asma bronquial, rinitis alérgica y eczema con estimadores de bienestar social ${ }^{8}$. Junto con el incremento de estas enfermedades, se ha producido una reducción a la exposición de agentes infecciosos en los primeros años de vida, secundaria a mejoría en las condiciones sanitarias medioambientales, hecho que alteraría el balance inmunológico hacia una respuesta predominantemente Th2, asociada a mayor producción de IgE y desarrollo de enfermedades alérgicas ${ }^{9,10}$. Sin embargo, los factores relacionados con condiciones de vida no son suficientes per se para explicar el aumento en la prevalencia del asma, existiendo controversia acerca del rol del medioambiente y de la alergia como determinantes de esta enferme$\operatorname{dad}^{11}$.

La población escolar presenta adecuadas características para estudiar el comportamiento de estas enfermedades, dada la facilidad en su acceso y la posibilidad de estudiar el efecto temprano a exposiciones ambientales. El capítulo chileno del estudio ISAAC (Internacional Study of Asthma and Allergy in Childhood) ${ }^{12}$ ayudó a cubrir un importante vacío de información existente en Chile, aportando además en el uso de instrumentos de medición estandarizados. En su primera fase, este estudio obtuvo información sobre la prevalencia y severidad del asma bronquial y enfermedades alérgicas. Chile participó incluyendo a niños entre 6-7 años y 13-14 años, en cuatro zonas geográficas (Santiago Centro y Sur, Valdivia y Punta Arenas), obteniéndose prevalencias de asma que oscilaron entre $16,5 \%$ y $20,5 \%$ en escolares de 6-7 años y 6,8\% a 11,7\% en escolares de 13-14 años ${ }^{13}$. Previamente un estudio midió la prevalencia de asma en escolares de 13-14 años del sector oriente de Santiago, obteniendo cifras mayores a las señaladas ${ }^{14}$.

El objetivo de este estudio fue analizar la influencia del nivel socioeconómico (NSE) en la prevalencia y severidad del asma (estimada mediante la frecuencia de episodios de sibilancias en el último año), utilizando un diseño transversal e instrumentos de recolección de información modificados del estudio ISAAC, para fines comparativos. Dado que posterior al estudio ISAAC se reportaron cambios en la prevalencia de asma en períodos breves de tiempo ${ }^{15}$, se incorporó como objetivo adicional evaluar la existencia de cambios en la prevalencia y síntomas de esta enfermedad en un período de 6 años, en similar área geográfica (Santiago Centro).

\section{MATERIAL Y MÉTODO}

Estudio de corte transversal, conducido entre noviembre y diciembre de 2000. En base a muestreo aleatorio simple de establecimientos educacionales de Santiago, se seleccionó una muestra de establecimientos públicos de la comuna de Santiago Centro y establecimientos particulares pagados de comunas del sector oriente (Las Condes, Vitacura y Providencia). La unidad de análisis comprendió a escolares de ambos sexos cursando $1^{\circ}$ ó $2^{\circ}$ año Básico (6-7 años) y $8^{\circ}$ Básico ó $1^{\circ}$ Medio (13-14 años). En base al Directorio de Establecimientos Educacionales se identificaron 103 establecimientos particulares pagados de las comunas mencionadas del sector oriente (matrícula de Enseñanza Básica de 43.877 alumnos y de Enseñanza Media de 22.891 alumnos). Por su parte, fueron identificados 40 establecimientos municipales de la comuna de Santiago Centro (matrícula en Enseñanza Básica de 17.096 alumnos y Enseñanza Media de 20.307 alumnos). La matrícula total de alumnos en los cursos correspondientes a las edades en estudio fue de 10.970 alumnos ( $1^{\mathrm{o}}$ y $2^{\mathrm{o}}$ Básico) y 11.227 alumnos ( $8^{\circ}$ Básico y $1^{\circ}$ Medio) en establecimientos del 
sector oriente y 3.432 alumnos ( $1^{\circ}$ y $2^{\circ}$ Básico) y 6.464 alumnos ( $8^{\circ}$ Básico y 1ํo Medio) en establecimientos del sector céntrico. Se utilizó el tipo de financiamiento y ubicación del establecimiento como estimador del NSE, correspondiendo los establecimientos del sector céntrico a NSE mediobajo y bajo y los del sector oriente a NSE alto. La relación entre el ingreso familiar autónomo y matrícula en el sistema escolar indica una asociación positiva entre ingreso y matrícula en establecimientos particulares pagados ${ }^{16}$.

El nivel de instrucción de los padres se estableció consignando los años declarados de estudios. Considerando el tamaño del grupo familiar y número de habitaciones del hogar, se elaboró un indicador de hacinamiento expresado en forma continua (personas/habitación) para independizar su lectura de una decisión de punto de corte. Para el cálculo del tamaño muestral se utilizó la recomendación del Steering Committee del estudio ISAAC, que sugiere reclutar idealmente 3.000 sujetos en cada estrato de edad para obtener el máximo de precisión, o de 1.000 escolares por grupo, magnitud que permite al menos el cálculo y comparación de prevalencias. Los procedimientos de muestreo y logística de la investigación fueron descritos en una publicación preliminar ${ }^{17}$. Los cuestionarios fueron completados en sus domicilios por padres o cuidadores de los niños de 6-7 años y por escolares del grupo de 13-14 años, bajo supervisión de sus profesores, quienes participaron en actividades de capacitación. Previamente fueron distribuidos instructivos a profesores y padres, señalando características generales de la enfermedad y formas de reconocer síntomas incluidos en el cuestionario. El cuestionario incluyó también una descripción simple de síntomas como disnea y sibilancias audibles.

Dado que en el periodo 1994-2000 el catastro de establecimientos presentó modificaciones, se utilizaron muestras independientes de colegios y estudiantes para la comparación de dicho periodo. El protocolo fue aprobado por el Comité de Ética de la Facultad de Medicina de la Pontificia Universidad Católica y contó con autorización de autoridades responsables de los establecimientos, requiriéndose del consentimiento informado de padres y apoderados.

Las principales variables dependientes analizadas fueron: diagnóstico de asma bronquial alguna vez en la vida (DA), sibilancias en los últimos 12 meses (SIB12) y frecuencia de los episodios de sibilancias (FES) (más de 4 episodios-sibilancias/ año), calculándose para cada grupo de edad prevalencias crudas. Las variables categóricas fueron comparadas mediante prueba de chi-cuadrado y los promedios mediante prueba t de Student. Intervalos de confianza para las prevalencias fueron calculados con 95\% de confianza (IC 95\%). Se utilizó análisis de regresión logística para calcular odds ratios (OR) de prevalencia. Las variables continuas fueron analizadas mediante regresión lineal simple y múltiple. Los modelos de regresión fueron ajustados por variables confundentes sugeridas en publicaciones del área (sexo, tabaquismo en el hogar, tamaño del grupo familiar, combustible utilizado para calefacción y cocina y nivel educacional de padres), incluyendo en los análisis preliminares una evaluación bivariada de dichas variables. La comparación entre el año 1994 y 2000 se efectuó mediante test de comparación de proporciones para muestras independientes. El tamaño muestral propuesto de al menos 1.000 escolares por grupo permite evaluar diferencias de hasta 5\%, con 95\% de confianza y $80 \%$ de potencia en la comparación de las prevalencias de síntomas. Para los cálculos se fijó un nivel de error de tipo I de 0,05, siendo utilizado el software estadístico STATA versión 8.0“.

\section{Resultados}

Un total de 7.864 cuestionarios fueron distribuidos, recuperándose 6.000 (76,3\%). Considerando las preguntas relacionadas con síntomas y diagnóstico de asma, fueron respondidos en forma completa 4.541 cuestionarios, 1.725 de Santiago Centro y 2.816 del área oriente de Santiago (2.253 mujeres y 2.288 varones). Se estimó una tasa global de participación de $72 \%$, siendo ésta de $75,5 \%$ en el grupo de menor edad y en establecimientos del sector céntrico. Las características de la muestra de escolares estudiada se resumen en la Tabla 1 . Se constataron diferencias en la frecuencia de variables relacionadas con el NSE entre los escolares de ambos tipos de establecimientos. La prevalencia cruda de DA para el grupo completo de escolares fue mayor en varones respecto de mujeres 
Tabla 1. Características generales de la muestra total de escolares estudiados, según ubicación del establecimiento educacional

\begin{tabular}{|c|c|c|c|}
\hline \multirow[t]{2}{*}{ Variables independientes } & \multicolumn{3}{|c|}{ Ubicación del establecimiento } \\
\hline & $\begin{array}{c}\text { Zona Centro } \\
(\mathrm{n}=1.725)\end{array}$ & $\begin{array}{l}\text { Zona Oriente } \\
(\mathrm{n}=\mathbf{2 . 8 1 6})\end{array}$ & $\mathbf{p}$ \\
\hline \multicolumn{4}{|l|}{ Sexo $(\%)$} \\
\hline Varones & 36,8 & 58,7 & \multirow[t]{2}{*}{$\mathrm{p}<0,001$} \\
\hline Mujeres & 63,2 & 41,3 & \\
\hline \multicolumn{4}{|l|}{ Instrucción materna (\%) } \\
\hline Sin instrucción/básica & 47,8 & 17,7 & \multirow[t]{3}{*}{$\mathrm{p}<0,001$} \\
\hline Enseñanza media & 35,9 & 18,2 & \\
\hline Universitaria & 16,7 & 64,1 & \\
\hline \multicolumn{4}{|l|}{ Instrucción paterna (\%) } \\
\hline Sin instrucción/básica & 45,3 & 11,2 & \multirow[t]{3}{*}{$\mathrm{p}<0,001$} \\
\hline Enseñanza media & 27,2 & 9,1 & \\
\hline Universitaria & 27,5 & 79,7 & \\
\hline Promedio grupo familiar & 4,7 & 5,3 & $\mathrm{p}<0,001$ \\
\hline \multicolumn{4}{|l|}{ Hacinamiento } \\
\hline (personas/dormitorio) & 1,8 & 1,3 & \multirow{2}{*}{$\mathrm{p}<0,001$} \\
\hline \multicolumn{3}{|l|}{ Combustible utilizado para calefacción (\%) } & \\
\hline Gas & 59,1 & 66,2 & \multirow[t]{5}{*}{$\mathrm{p}<0,001$} \\
\hline Electricidad & 5,5 & 5,2 & \\
\hline Leña & 0,2 & 5,1 & \\
\hline Parafina & 32,5 & 8,5 & \\
\hline Petróleo & 0,5 & 13,9 & \\
\hline \multicolumn{4}{|l|}{ Combustible utilizado para cocinar (\%) } \\
\hline Gas & 99,4 & 97,3 & \multirow[t]{3}{*}{$\mathrm{p}<0,001$} \\
\hline Electricidad & 0,3 & 1,9 & \\
\hline Otro & 0,3 & 0,8 & \\
\hline \multicolumn{4}{|l|}{ Piso de la vivienda (\%) } \\
\hline Madera/parquet & 33,4 & 26,1 & \multirow[t]{4}{*}{$\mathrm{p}<0,001$} \\
\hline Baldosa & 17,3 & 7,5 & \\
\hline Linóleo/flexit & 23,6 & 0,7 & \\
\hline Alfombra/cubrepiso & 14,7 & 63,3 & \\
\hline Exposición ambiental a humo de tabaco (\%) & 54,2 & 50,0 & $\mathrm{p}=0,006$ \\
\hline Promedio fumadores/hogar & 1,73 & 1,70 & $\mathrm{p}=0,21$ \\
\hline Mascotas en hogar (\%) & 63,3 & 57,6 & $\mathrm{p}<0,001$ \\
\hline
\end{tabular}

(13,2\%; IC 95\%: 11,8\%-14,6\% y 10,8\%; IC 95\%: 9,5\%-12,2\%, respectivamente). La prevalencia de DA fue estadísticamente mayor en escolares de 13-14 años (13,8\%: IC 95\%: 12,3\%-15,2\%) respecto del grupo de 6-7 años (10,1\%; IC 95\%: $8,7 \%-11,3 \%) .\left(\mathrm{p}<10^{-4}\right)$.

La asociación entre las variables DA, SIB12 y FES con las principales variables de exposición se resumen en la Tabla 2. En términos de edad, los varones de 6-7 años presentaron una prevalencia significativamente mayor de FES comparados con mujeres del mismo grupo etario (3,3\% vs $1,9 \%$ ), mientras que los varones entre 13-14 años presentaron una prevalencia significativamente mayor de DA en comparación con mujeres de igual edad (16,5\% vs $12,0 \%)$. Con respecto a la ubicación del estableci- 
Tabla 2. Prevalencia porcentual de síntomas respiratorios seleccionados según variables de exposición, en escolares de $1^{\circ}-2^{\circ}$ Básico y $8^{\circ}$ Básico- $1^{\circ}$ medio

\begin{tabular}{|c|c|c|c|c|c|c|}
\hline \multirow[b]{3}{*}{ Variables } & \multicolumn{6}{|c|}{ Grupo de edad } \\
\hline & \multicolumn{3}{|c|}{$\begin{array}{c}\text { 6-7años } \\
(\mathrm{n}=2.254)\end{array}$} & \multicolumn{3}{|c|}{$\begin{array}{c}13-14 \text { años } \\
(\mathrm{n}=2.279)\end{array}$} \\
\hline & DA & SB12 & FES & DA & SB12 & FES \\
\hline \multicolumn{7}{|l|}{ Sexo } \\
\hline Varones & 10,5 & 16,6 & $3,3(\mathbb{\$})$ & $16,5(\$)$ & 11,0 & 1,5 \\
\hline Mujeres & 9,4 & 14,5 & 1,9 & 12,0 & 11,2 & 2,5 \\
\hline \multicolumn{7}{|c|}{ Ubicación del establecimiento } \\
\hline Central & 8,8 & $21,9(\sqrt{(})$ & $4,5(\$)$ & $12,2(\S)$ & $12,6(\$)$ & 1,9 \\
\hline Oriente & 10,7 & 12,9 & 1,8 & 15,2 & 9,7 & 1,9 \\
\hline \multicolumn{7}{|l|}{ Piso de la vivienda } \\
\hline Madera/parquet & 8,4 & $14,6(\$)$ & 2,9 & 11,7 & 10,0 & 1,7 \\
\hline Baldosa & 12,2 & 21,9 & 4,9 & 15,0 & 11,5 & 2,8 \\
\hline Linóleo/flexit & 10,2 & 27,4 & 1,9 & 12,0 & 13,5 & 1,1 \\
\hline Alfombra/cubrepiso & 11,3 & 13,3 & 2,1 & 15,1 & 10,8 & 2,1 \\
\hline \multicolumn{7}{|c|}{ Exposición ambiental a humo de tabaco } \\
\hline Sí & 10,2 & 17,2 & 2,7 & 14,6 & $12,3(\$)$ & 2,0 \\
\hline No & 10,0 & 14,5 & 2,7 & 13,1 & 9,4 & 1,7 \\
\hline \multicolumn{7}{|c|}{ Nivel de instrucción materna } \\
\hline Sin instrucción, básica & 8,5 & $18,0(\$)$ & 3,9 & 14,2 & $13,7(\$)$ & 2,2 \\
\hline Enseñanza media & 11,8 & 18,2 & 2,7 & 11,7 & 10,7 & 1,2 \\
\hline Universitaria & 10,2 & 13,2 & 2,0 & 14,8 & 9,4 & 2,3 \\
\hline \multicolumn{7}{|l|}{ Nivel de instrucción paterna } \\
\hline Sin Instrucción, básica & $7,4(\wp)$ & $19,5(\S)$ & 3,5 & 14,1 & 12,6 & 1,9 \\
\hline Enseñanza media & 10,1 & 15,3 & 3,1 & 11,6 & 11,1 & 1,0 \\
\hline Universitaria & 11,2 & 14,4 & 2,3 & 14,4 & 10,4 & 2,3 \\
\hline \multicolumn{7}{|l|}{ Mascotas en hogar } \\
\hline Sí & 9,8 & 15,7 & 2,7 & 14,0 & 10,5 & 1,6 \\
\hline No & 10,3 & 15,8 & 2,7 & 13,3 & 12,0 & 2,7 \\
\hline
\end{tabular}

DA =diagnóstico de asma; SB12 =sibilancias últimos 12 meses; FES ( $<4$ episodios-sibilancias/año) $\mathfrak{S}=\mathrm{p}<0,05$

miento, solamente en escolares de 13-14 años se encontró asociación significativa entre esta variable y DA, siendo mayor en el sector oriente $(15,2 \%$ vs 12,2\%). En ambos grupos de edad se encontró asociación significativa entre la ubicación del establecimiento y reporte de SIB12, siendo mayores las prevalencias en Santiago Centro (21,9\% vs $12,9 \%$ y $12,6 \%$ vs $9,7 \%)$. Respecto a FES, únicamente en escolares de 6-7 años de Santiago Centro se verificó una prevalencia significativamente mayor que aquellos del sector oriente ( $4,5 \%$ vs $1,8 \%$ ).
En relación al nivel educacional de los progenitores, la prevalencia de DA en escolares de 6-7 años fue significativamente menor en niños cuyos padres varones no reportan instrucción o bien sólo cursaron enseñanza básica, mientras que la prevalencia de SIB12 fue significativamente mayor cuando ambos padres presentaban dicho nivel educacional. Esta asociación fue estadísticamente significativa también para escolares de 13-14 años, aunque sólo para grados menores de instrucción materna. 
No se encontraron diferencias significativas entre frecuencia de síntomas y combustible utilizado para cocina y calefacción en el hogar. Se encontró asociación inversa entre nivel de hacinamiento y prevalencia de DA (coeficiente $-0,03$; $p$ $=0,026)$ y asociación directa con SIB12 (coeficiente $+0,03 ; \mathrm{p}=0,022$ ). La prevalencia de SIB12 en escolares de 6-7 años fue significativamente menor en hogares con cubrepisos o alfombras. La exposición ambiental a humo del tabaco se asoció significativamente con mayor prevalencia SIB12 en el grupo de adolescentes (12,3\% vs 9,4\%).

Las Tablas 3 y 4 resumen el riesgo ajustado de presentar DA, SIB12 y FES según la exposición a diversas variables independientes. El DA fue más prevalente en varones de 13-14 años (OR ajustado $=1,4$; IC 95\%; 1,01-1,80) respecto de mujeres de similar edad. En ambos grupos de edad se observó menor riesgo de SIB12 en escolares del sector oriente en comparación a los de Santiago
Centro (OR ajustado =0,5; IC 95\%; 0,4-0,7 y OR ajustado $=0,7$; IC 95\%; 0,6-0,9), mientras que el riesgo de FES fue significativamente menor en escolares del sector oriente únicamente en el grupo de niños de 6-7 años (OR ajustado $=0,4$; IC $95 \%$ 0,2-0,7). En ambos grupos de edad, un mayor nivel de instrucción de ambos padres se asoció con reducción significativa en la prevalencia de SIB12. En cuanto a FES, un mayor nivel de instrucción materna resultó asociado con menor riesgo de asma severa solamente en escolares de 6-7 años, sin diferencias asociadas al nivel educacional del padre. Por su parte, un mayor nivel de instrucción paterna determinó un incremento de $60 \%$ en el DA (OR ajustado $=1,6 ; 95 \%$ IC; $1,1-2,3$ ) en escolares de 6-7 años, sin encontrarse diferencias significativas en el DA según el nivel educacional de la madre.

El análisis comparativo entre los años 1994 y 2000 (restringido a escolares de establecimientos

Tabla 3. 0 dds ratios de prevalencia crudos y ajustados para síntomas respiratorios en el grupo de escolares de $1^{\circ}-2^{\circ}$ Básico

\begin{tabular}{|c|c|c|c|c|c|c|}
\hline \multirow[t]{2}{*}{ Variable } & \multicolumn{2}{|c|}{ DA } & \multicolumn{2}{|c|}{ SIB12 } & \multicolumn{2}{|c|}{ FES } \\
\hline & ORC & ORA & ORC & ORA & ORC & ORA \\
\hline \multicolumn{7}{|l|}{ Sexo $(*)$} \\
\hline Mujeres (referencia) & 1 & 1 & 1 & 1 & 1 & 1 \\
\hline Varones & 1,1 & 1,1 & 1,2 & 1,2 & 1,8 & 1,6 \\
\hline \multicolumn{7}{|l|}{ Establecimiento $(\dagger)$} \\
\hline Central (referencia) & 1 & 1 & 1 & 1 & 1 & 1 \\
\hline Oriente & 1,2 & 1,2 & $0,5(\$)$ & $0,5(\mathfrak{S})$ & $0,4(\S)$ & $0,4(\S)$ \\
\hline \multicolumn{7}{|l|}{ Educación madre $\left({ }^{\ddagger}\right)$} \\
\hline Sin/básica (referencia) & 1 & 1 & 1 & 1 & 1 & 1 \\
\hline Media & 1,4 & 1,4 & 1 & 0,9 & 0,7 & 0,7 \\
\hline Universitaria & 1,2 & 1,2 & $0,7(\$)$ & $0,7(\mathfrak{S})$ & $0,5(\$)$ & $0,5(\$)$ \\
\hline \multicolumn{7}{|l|}{ Educación padre $\left({ }^{\ddagger}\right)$} \\
\hline Sin/básica (referencia) & 1 & 1 & 1 & 1 & 1 & 1 \\
\hline Media & 1,4 & 1,4 & 0,7 & 0,7 & 0,9 & 0,9 \\
\hline Universitaria & $1,6(\$)$ & $1,6(\$)$ & $0,7(\$)$ & $0,7(\sqrt{(})$ & 0,6 & 0,6 \\
\hline
\end{tabular}

DA =diagnóstico de asma; SB12 =sibilancias últimos 12 meses; FES ( $<4$ episodios-sibilancias/año) ORC $=$ odds ratio crudo; ORA $=$ odds ratio ajustado

(*) OR ajustado por ubicación del establecimiento educacional, mascotas en el hogar y exposición ambiental a humo de tabaco.

(†) OR ajustado por sexo, educación de los padres y exposición ambiental a humo de tabaco.

$\left.{ }^{\ddagger}\right)$ OR ajustado por sexo, mascotas en el hogar y exposición ambiental a humo de tabaco.

(\$) Diferencia estadísticamente significativa. 
Tabla 4. 0 dds ratios de prevalencia crudos y ajustados para síntomas respiratorios en el grupo de escolares de $8^{\circ}$ Básico- $1^{\circ}$ M edio

\begin{tabular}{|c|c|c|c|c|c|c|}
\hline \multirow[t]{2}{*}{ Variable } & \multicolumn{2}{|c|}{ DA } & \multicolumn{2}{|c|}{ SIB12 } & \multicolumn{2}{|c|}{ FES } \\
\hline & ORC & ORA & ORC & ORA & ORC & ORA \\
\hline \multicolumn{7}{|l|}{$\operatorname{Sexo}(*)$} \\
\hline Mujeres (referencia) & 1 & 1 & 1 & 1 & 1 & 1 \\
\hline Varones & $1,4(\$)$ & $1,4(\$)$ & 1 & 1,4 & 1,6 & 1,8 \\
\hline \multicolumn{7}{|l|}{ Establecimiento $\left({ }^{\dagger}\right)$} \\
\hline Central (referencia) & 1 & 1 & 1 & 1 & 1 & 1 \\
\hline Oriente & $1,3(\$)$ & $1,3(\$)$ & $0,7(\$)$ & $0,7(\$)$ & 1 & 1 \\
\hline \multicolumn{7}{|l|}{ Educación madre $\left({ }^{\ddagger}\right)$} \\
\hline Sin/básica (referencia) & 1 & 1 & 1 & 1 & 1 & 1 \\
\hline Media & 0,8 & 0,8 & 0,8 & 0,8 & 0,5 & 0,5 \\
\hline Universitaria & 1 & 1 & $0,7(\$)$ & $0,6(\$)$ & 1 & 1 \\
\hline \multicolumn{7}{|l|}{ Educación padre $\left({ }^{\ddagger}\right)$} \\
\hline Sin/básica (referencia) & 1 & 1 & 1 & 1 & 1 & 1 \\
\hline Media & 0,8 & 0,8 & 0,9 & 0,9 & 0,5 & 0,5 \\
\hline Universitaria & 1 & 0,9 & 0,8 & $0,8(\$)$ & 1,2 & 1,1 \\
\hline
\end{tabular}

DA =diagnóstico de asma; SB12 =sibilancias últimos 12 meses; FES ( $<4$ episodios-sibilancias/año) ORC =odds ratio crudo; ORA =odds ratio ajustado

$\left(^{*}\right)$ OR ajustado por ubicación del establecimiento educacional, mascotas en el hogar y exposición ambiental a humo de tabaco.

(†) OR ajustado por sexo, educación de los padres y exposición ambiental a humo de tabaco.

(‡) OR ajustado por sexo, mascotas en el hogar y exposición ambiental a humo de tabaco.

( $($ ) Diferencia estadísticamente significativa.

del sector céntrico) mostró en niños de 6-7 años aumento estadísticamente significativo en la prevalencia de SIB12 y FES, no así en DA (Figura 1). En escolares de 13-14 años la prevalencia de las tres variables dependientes no experimentó variaciones significativas durante igual periodo (Figura 2).

\section{DisCUSIÓN}

En escolares de 6-7 años se observó una relación inversa entre algunas de las variables estimadoras de NSE y la prevalencia de SIB12 y FES, siendo ambas prevalencias mayores en escolares de menor NSE. Esto concuerda con lo comunicado por Corvalán et al en Limache en población adulta joven, quienes encuentran asociación inversa entre diferentes estimadores del NSE y síntomas de asma e hiperreactividad bronquial ${ }^{18}$. Hallazgos similares en un análisis de tendencia encuentra von Mutius ${ }^{7}$, al constatar aumento significativo en la prevalencia de fiebre del heno y sensibilización atópica en una cohorte de niños alemanes orientales de 5 a 9 años estudiados al momento de la unificación alemana y cuatro años más tarde (1995-1996). Por el contrario, en niños alemanes occidentales de 9 a 11 años, la prevalencia se mantuvo estable entre 1992-200119. La mayor prevalencia de DA en adolescentes de 13-14 años de sectores más acomodados concuerda con lo publicado. Esto podría explicarse, al menos en este grupo de edad, por mejor acceso a atención médica y, por tanto, mayor probabilidad de ser diagnosticado como asmático. Por su parte, la prevalencia de SIB12 fue mayor en el grupo de menor NSE, en ambos grupos de edad. Esto se explicaría por el hecho de que la expresión sintomática de la enfermedad es independiente 


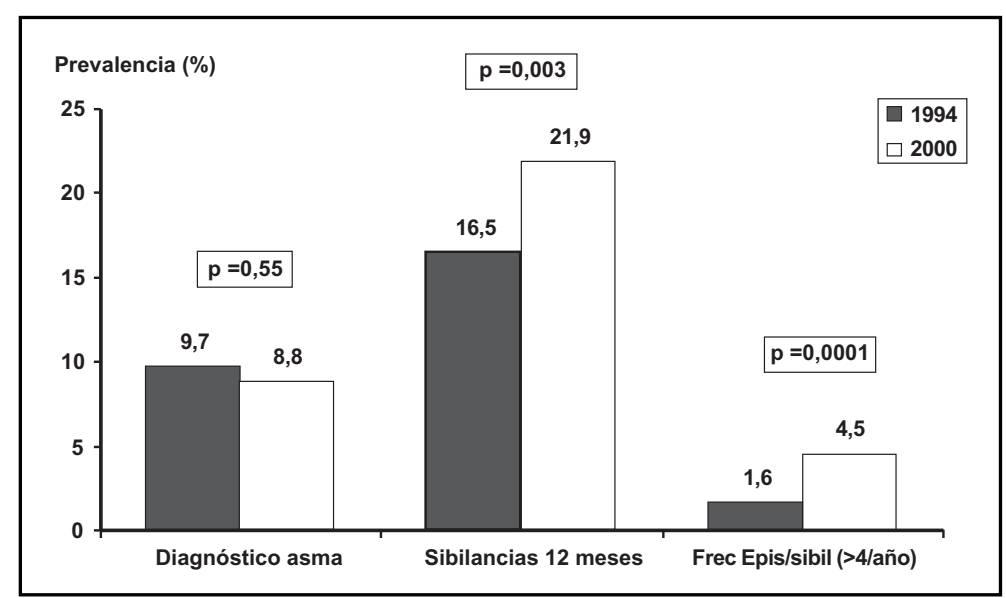

Figura 1. Prevalencia de diagnóstico de asma, sibilancias en los últimos 12 meses y frecuencia de episodios de sibilancias en los años 1994 y 2000 en escolares de $1^{\circ}$ y 2oásico (Santiago Centro).

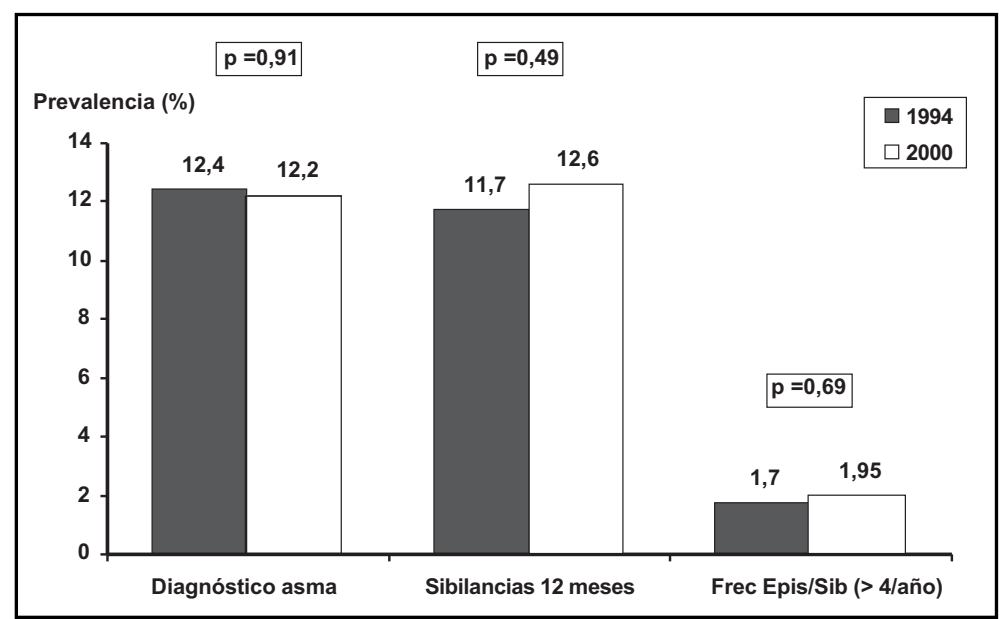

Figura 2. Prevalencia de diagnóstico de asma, sibilancias en los últimos 12 meses y frecuencia de episodios de sibilancias en los años 1994 y 2000 en escolares de $8^{\circ}$ Básico-1º Medio (Santiago Centro).

del DA, pudiendo estar condicionada por factores de riesgo más prevalentes en NSE más bajos (exposición a ambientes más contaminados ${ }^{20,21}$, menor adherencia a terapias ${ }^{22}$, mal manejo de crisis asmáticas $^{23}$, inadecuada comunicación con médicos tratantes y menor conocimiento sobre la patología ${ }^{24-26}$ y diferente percepción de los síntomas de la enfermedad ${ }^{18}$ ).

En Chile las consultas por síndrome bronquial obstructivo en el nivel primario de atención constituyen 23\% del total de consultas por morbi- lidad aguda en menores de 15 años ${ }^{27}$, correspondiendo a $16 \%$ de las consultas entre 5 y 15 años ${ }^{28}$. Al cumplir el año de edad, 43,1\% de los lactantes han presentado sibilancias recurrentes 25 . La prevalencia de asma en Chile está comparativamente en situación intermedia ${ }^{8}$, sin embargo, la mortalidad en menores de 20 años en Chile ha declinado, a diferencia de lo observado en Estados Unidos de Norteamérica. Las hospitalizaciones por asma en la infancia son infrecuentes, dando cuenta de $0,3 \%$ y $0,4 \%$ de los egresos hospitalarios en 
menores de 20 y 10 años, respectivamente ${ }^{28}$. El Estudio de Carga de Enfermedad (1993) ubicó el asma bronquial en 6 o lugar como causa de AVISA (años de vida perdidos ajustados por discapacidad) en ambos sexos, generando una pérdida anual de 55.118 AVISA $^{29}$.

El presente trabajo presenta algunas limitaciones metodológicas. Su diseño transversal limita la proposición de relaciones causales y el control exhaustivo de variables confundentes, pudiendo verse afectado por el efecto de "causalidad reversa". Posiblemente esta limitación pueda, en parte, explicar el hallazgo de menor prevalencia de SIB12 en hogares con cubrepisos o alfombras. La medición del NSE es compleja y en su caracterización no siempre se logra integrar sus múltiples componentes ${ }^{18,23,25}$, los cuales incluso pueden actuar en sentidos opuestos o con diferente magnitud en el asma ${ }^{18,23}$. Por esta razón en el presente estudio se utilizaron diferentes aproximaciones para su estimación, obteniéndose resultados concordantes en el análisis de estas dimensiones con los estimadores de la enfermedad. El uso de cuestionarios en investigación epidemiológica del asma es una forma adecuada para la evaluación poblacional de síntomas de asma, y su estandarización permite efectuar adecuadas comparaciones en aplicaciones separadas en el tiempo $13,15,37,38$.

La tasa de no participación en el estudio fue de $28 \%$, desconociéndose las motivaciones y características individuales de los escolares no participantes. No obstante, los porcentajes de participación de los colegios según su ubicación y la concentración del estudio en meses de verano reducen en parte la probabilidad de ocurrencia de sesgo de selección.

La contaminación del aire está asociada con modificación en la frecuencia de síntomas de la enfermedad y por tanto, puede incidir en la prevalencia de ellos ${ }^{23}$. Aun cuando esta variable no fue incluida en el presente protocolo, durante el trabajo de campo no se registraron episodios de emergencia o críticos en la calidad del aire. En el periodo 1994-2000 se observó mejoría sostenida en los patrones de calidad del aire, siendo poco probable que esta variable pudiera explicar los cambios observados ${ }^{39}$.

En los últimos años, diversas investigaciones han agregado información sobre la influencia de nuevas variables en el asma, tales como factores nutricionales, perinatales, ambientales y familiares. Esta información resulta relevante a la luz de investigaciones que han establecido una base hereditaria del asma ${ }^{30}$, la existencia de asociación con obesidad ${ }^{31-33}$, con bajo peso de nacimiento ${ }^{34}$ y con contaminación ambiental ${ }^{35}$. Los mecanismos con que operan estos factores no son plenamente conocidos, pudiendo ser expresión de efectos de confusión e interacción epidemiológica o, simplemente, asociaciones espúreas. Considerando que Chile está en una fase avanzada de transición epidemiológica, se espera una modificación en la frecuencia y comportamiento de enfermedades con base inmunológica, entre las cuales se incluye el asma bronquial ${ }^{9,36}$.

Finalmente, se constató un incremento significativo en SIB12 y FES entre 1994 y 2000 en escolares de 6-7 años asistentes a establecimientos educacionales del sector céntrico de Santiago, situación que no se verificó en el grupo de escolares entre 13-14 años. Esta tendencia concuerda con el reporte del grupo ISAAC, el cual demostró aumento de la prevalencia de asma en el grupo de escolares entre 6-7 años, reconociendo que existe un patrón variable de cambio en las prevalencias, predominando los incrementos por sobre los descensos ${ }^{37}$. La ausencia de un patrón homogéneo de cambios en la prevalencia explica las diferencias entre los resultados de nuestro estudio y aquellos reportados por Mallol ${ }^{38}$, quien verificó en Santiago un descenso de la prevalencia de "asma alguna vez", "sibilancias en los últimos 12 meses" y "episodio severo" al comparar 3.182 niños de 6-7 años encuestados en 1994 con 3.075 niños de igual edad encuestados en 2002. Al respecto, concordamos con su reflexión en cuanto a que es posible que los aumentos y disminuciones de las prevalencias alternen en el tiempo, guardando relación con eventos ecológicos concomitantes.

En síntesis y en concordancia con una comunicación previa en relación con rinitis alérgica en escolares $^{17}$, este estudio apoya la hipótesis acerca de la influencia de variables relacionadas con el NSE en el DA, SIB12 y FES en escolares de la Región Metropolitana de Santiago. Adicionalmente, se constató un incremento significativo en la prevalencia de SIB12 y FES entre los años 1994 y 2000 en escolares de 6-7 años asistentes a estable- 
cimientos educacionales del sector céntrico de Santiago.

\section{Agradecimientos}

A las autoridades de los establecimientos educacionales que prestaron su valiosa colaboración para la realización de este estudio: Verbo Divino, SS.CC Manquehue, San Benito, San Pedro Nolasco, SS.CC Apo-

\section{REFERENCIAS}

1. Eder W, Ege M, Von Mutius E. The asthma epidemics. N Engl J Med 2006; 355: 2226-35.

2. Carrasco E. Epidemiology of asthma in Latin American children. Chest 1987; 91: 93S-97S.

3. Camargos P, Castro R, Feldman J. Prevalencia de síntomas relacionados con el asma en escolares de Campos Gerais (MG), Brasil. Rev Panam Salud Publica 1999; 6: 8-14.

4. Guimaráes A, Couto C, Aires V. Prevalência de sintomas respiratórios e condiçoes clínicas associadas á asma em escolares de 6 a 14 anos no Rio de Janeiro. Rev Assoc Med Bras 2002; 48: 54-9.

5. Valenzuela P, Gómez G, Galleguillos F. Prevalencia del asma bronquial en escolares de Santiago, Chile. Rev Med Chile 1981; 109: 259-66.

6. Toro J, Carrasco E, Reyes M. Asma bronquial: incremento en otros países, ¿incremento en Chile? Bol Epidemiol Chile 1987; 15: 129-38.

7. Von Mutius E, Weiland S, Fritzsch C, Duhme H, Keil U. Increasing prevalence of hay fever and atopy among children in Leipzig, East Germany. The Lancet 1998; 351: 862-6.

8. Stewart A, Mitchell E, Pearce N, Strachan D, Weiland S. The relationship of per capita gross national product to the prevalence of symptoms of asthma and other atopic diseases in children (ISAAC). Int J Epidemiol 2001; 30: 173-9.

9. Luque C, Cisternas F, Araya M. Cambios del patrón de enfermedad en la postransición epidemiológica en salud en Chile, 1950-2003. Rev Med Chile 2006; 134: 703-12.

10. Illi S, Von Mutius E, Lau S, Bergmann R, Niggemann B, Sommerfeld C ET AL. Early childhood infectious diseases and the development of asthma up to school age: a birth cohort study. BMJ 2001; 322: 390-5.

11. Borchers AT, Keen CL, Gershwin ME. Hope for the hygiene hypothesis: when the dirt hits the fan. J Asthma 2005; 42: 225-47. quindo, Scuola Italiana, Compañía de María Apoquindo, SEK, Bradford School, Dunalastair, Redland School, La Maisonnette, San Ignacio El Bosque, Lo Castillo, Wenlock School, British High School, Francisco Olea, Guillermo Matta, INSUCO, Isaura Dinator de Guzmán, Benjamín Vicuña Mackenna, Elvira Errázuriz, Miguel de Cervantes y Saavedra, Cambridge School, Blas Cañas, Francisco Arriarán, Cadete Arturo Prat Chacón, y Liceo № 1 .
12. Beasley R, Keil U, Von Mutius E, Pearce N. Worldwide variation in prevalence of symptoms of asthma, allergic rhinoconjuntivitis, and atopic eczema: ISAAC. The Lancet 1998; 351: 1225-32.

13. Mallol J, Cortez E, Amarales L, Sanchez I, Calvo G, Soto S et al. Prevalencia de asma en escolares chilenos. Estudio descriptivo de 24.470 niños. ISAAC-Chile. Rev Med Chile 2000; 128: 279-85.

14. Carrasco E, Velasco C, Sepúlveda R, Vicherat L, Feijoo R, Rodriguez MA et al. Prevalencia de asma en escolares de 13-15 años del sector oriente de Santiago. Rev Chil Enf Respir 2000; 16: 128-41.

15. Maziak W, Behrens T, Brasky TM, Duhme H, Rzehak P, WeILAND SK ET AL. Are asthma and allergies in children and adolescents increasing? Results from ISAAC phase I and phase III surveys in Münster, Germany. Allergy 2003; 58: 572-9.

16. SApeli C, Torche A. Subsidios al alumno o a la escuela: efectos sobre la elección de colegios públicos. Cuadernos de Economía 2002; 117: 175-202.

17. Caussade S, Valdivia G, Navarro H, Pérez E, Aquevedo A, Sánchez I. Prevalencia de síntomas de rinitis alérgica y su relación con factores de riesgo en escolares de Santiago, Chile. Rev Med Chile 2006; 134: 456-64.

18. Corvalán C, Amigo H, Bustos P, Rona R. Socioeconomics Risk factors for asthma in Chilean young adults. Am J Public Health 2005; 95: 1375-81.

19. Zollner IK, Weiland SK, Piechotowski I, Gabrio T, Von Mutius E, Link B ET AL. No increase in the prevalence of asthma, allergies, and atopic sensitisation among children in Germany: 1992-2001. Thorax 2005; 60: 545-8.

20. Rosenstreich DL, Eggleston P, Kattan M, Baker D, Slavin RG, Gergen P et al. The role of cockroach allergy and exposure to cockroach allergen in causing morbidity among inner-city children with asthma. N Engl J Med 1997; 336: 1356-63.

21. Burr ML, Verrall C, Kaur B. Social deprivation and asthma. Respir Med 1997; 91: 603-8. 
22. LANG DM, Sherman MS, Polansky M. Guidelines and realities of asthma management. The Philadelphia Story. Arch Intern Med 1997; 157: 1193-2000.

23. Hanania NA, David-Wang A, Kesten S, Chapman KR. Factors associated with emergency department dependence of patients with asthma. Chest 1997; 111: 290-5.

24. Rona R. Asthma and poverty. Thorax 2000; 55: 23944.

25. Mallol J, Andrade R, Auger F, Rodríguez J, Alvarado $\mathrm{R}$, Figueroa L. Wheezing during the first year of life in infants from low-income population: a descriptive study. Allergol Immunopathol 2005; 33: 257-63.

26. Mallol J. Childhood asthma in developing countries. Low income aspects and related matters. Allergol Immunopathol 2000; 28: 283-6.

27. Aranda C, Astudillo P, Mancilla P, Caussade S, Girardi G. Caracterización epidemiológica de las consultas pediátricas por causa respiratoria en atención primaria en Chile. OPS, Serie HCT/AIEPI-3E 1998; I: 43-9.

28. Astudillo P. Asma infantil en Chile. Neumología Pediátrica 2006; 1: 91-3.

29. MINSAL-Chile. Estudio Carga de Enfermedad en Chile 1996. Disponible en www.minsal.cl [consultado en $01 / 01 / 2007$.

30. HaLl IP. Genetics and pulmonary medicine: asthma. Thorax 1999; 54: 65-9.

31. Ford ES. The epidemiology of obesity and asthma. J Allergy Clin Immunol 2005; 115: 897-909.

32. Castro-Rodríguez J, Holberg C, Morgan W, Wright A, Martinez F. Increased incidence of asthma like symptoms in girls who become overweight or obese during the school years. Am J Respir Crit Care Med 2001; 163: 1344-9.

33. Huang SL, Shiao GM, Chou P. Association between body mass index and allergy in teenage girls in Taiwan. Clin Exp Allergy 1999; 29: 323-9.

34. Nepomnyaschy L, Reichman NE. Low birthweight and asthma among young urban children. Am J Public Health 2006; 96: 1604-10.

35. OYARZún M. Contaminación atmosférica y asma bronquial. Rev Chil Enf Respir 2000; 16: 142-7.

36. VAldivia G. Transición epidemiológica: la otra cara de la moneda. Rev Med Chile 2006; 134: 675-8.

37. Asher Mi, Montefort S, Bjorksten B, Lai CKW, Stranchan DP, Weiland SK, Williams H and the ISAAC Phase Three Study Group. Worldwide time trends in the prevalence of symptoms of asthma, allergic rhinoconjunctivitis, and eczema in childhood: ISAAC Phases One and Three repeat multicountry crosssectional surveys. Lancet 2006; 368: 733-43.

38. Mallol J, Aguirre V, Aguilar P, Calvo M, Amarales L, Arellano P et al. Cambios en la prevalencia de asma en escolares chilenos entre 1994 y 2002: International Study of Asthma and Allergies in Childhood (ISAAC) - Chile phases I and III. Rev Med Chile 2007; 135: 580-6.

39. Evolución de la calidad del aire en Santiago 1997 2003. Comisión Nacional del medio ambiente (CONAMA). Disponible en: http: //www.conama.cl/rm/ 568/articles-29215_pdf.pdf. Consultado el 02 julio de 2008. 